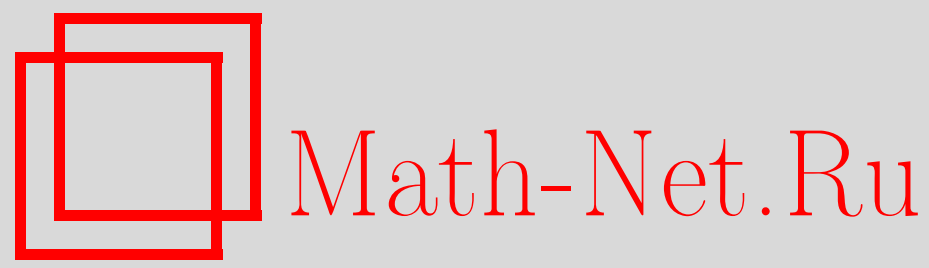

М. И. Дьяченко, Равномерная сходимость двойных рядов Фурье для классов функций с анизотропной гладкостью, Матем. заметки, 1996, том 59, выпуск 6, 937-943

DOI: https://doi.org/10.4213/mzm1795

Использование Общероссийского математического портала MathNet.Ru подразумевает, что вы прочитали и согласны с пользовательским соглашением

http://www . mathnet.ru/rus/agreement

Параметры загрузки:

IP : 54.224 .60 .19

26 апреля 2023 г., $17: 37: 22$

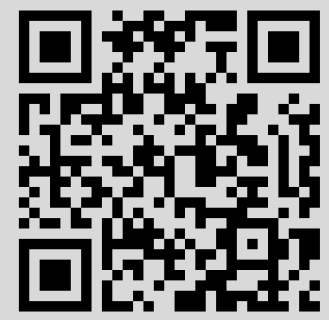




\section{РАВНОМЕРНАЯ СХОДИМОСТЬ ДВОЙНЫХ РЯДОВ ФУРЬЕ ДЛЯ КЛАССОВ ФУНКЦИЙ С АНИЗОТРОПНОЙ ГЛАДКОСТЬЮ}

\section{М. И. Дьяченко}

1. Введение. Всюду ниже будут рассматриваться $2 \pi$-периодические по каждой переменной функции двух переменных $f(x, y) \in C\left(T^{2}\right)$, где $T=[-\pi, \pi)$. Как обычно, положим $\|f\|=\sup _{(x, y) \in T^{2}}|f(x, y)|$ и пусть

$$
\sum_{m, n \in \mathbb{Z}} a_{m, n} e^{i m x} e^{i n y}
$$

- это двойной тригонометрический ряд Фурье функции $f(x, y)$.

Полным модулем непрерывности функции $f(x, y)$ будем называть выражение

$$
\omega(f, \delta)=\sup _{|(h, t)| \leqslant \delta}\|f(x+h, y+t)-f(x, y)\|
$$

при $\delta \in(0,2 \pi)$, частными модулями непрерьвности по переменньм $x$ и $y$ соответственно выражения

$$
\omega_{1}(f, \delta)=\sup _{|h| \leqslant \delta}\|f(x+h, y)-f(x, y)\| \quad \text { и } \quad \omega_{2}(f, \delta)=\sup _{|t| \leqslant \delta}\|f(x, y+t)-f(x, y)\|
$$

при $\delta \in(0,2 \pi)$ и, наконец, смешанньм модулем непрерьвности - выражение

$$
\omega\left(f, \delta_{1}, \delta_{2}\right)=\sup _{|h| \leqslant \delta_{1},|t| \leqslant \delta_{2}}\|f(x+h, y+t)-f(x+h, y)-f(x, y+t)+f(x, y)\|
$$

при $\delta_{1}, \delta_{2} \in(0,2 \pi)$. Через $C$ ниже будут обозначаться положительные абсолютные постоянные, через $C(\omega)$ - положительные постоянные, зависящие лишш от $\omega$ и т.д. Если $a$ - число, то через а будем обозначать двумерный вектор, обе координаты которого равны $a$.

Существует много различных определений сходимости двойных числовых рядов. Наиболее хорошо изучены сходимость по Прингсхейму, по квадратам и по кругам. Ситуация для двойных функциональных рядов и, в частности, для двойных рядов Фурье совершенно аналогичная. В настоящей статье речь будет идти только о равномерной сходимости ряда (1).

Отметим, что в статье [1] Л. В. Жижиашвили были установлены такие результаты (мы формулируем теоремы только для двумерного случая).

Теорема А. Если функи, ия $f(x, y) \in C\left(T^{2}\right) u \omega(f, \delta)=o\left((\ln (1 / \delta))^{-2}\right) n p u$ $\delta \rightarrow+0$, то ряд Фурье функиии $f(x, y)$ равномерно сходится $\kappa$ ней.

Работа выполнена при финансовой поддержке Международного научного фонда, грант NCJ000. 
Tеорема Б. Существует функиия $f(x, y) \in C\left(T^{2}\right)$, maкая что $\omega(f, \delta)=$ $O\left((\ln (1 / \delta))^{-2}\right)$ при $\delta \rightarrow+0$, но ряд Фурье функции $f(x, y)$ расходится по квадратам в метрике $C\left(T^{2}\right)$.

Что же касается сходимости по кругам, то здесь Ш.А. Алимовым и В.А. Ильиным [2] была доказана такая теорема.

Tеорема В. Если функиия $f(x, y) \in C\left(T^{2}\right) u \omega(f, \delta)=O\left(\delta^{1 / 2}\right) n p u \delta \rightarrow+0$, то ряд Фурье функиии $f(x, y)$ равномерно сходится по кругам. При этом условие $\omega(f, \delta)=O\left(\delta^{1 / 2-\varepsilon}\right)$ при $\delta \rightarrow+0$, где некоторое $\varepsilon>0$, уже не обеспечивает такой сходимости.

Уже из этих результатов видно, что теоремы для различных видов сходимости носят совершенно разный характер. В связи с этим представляет интерес нахождение условий, обеспечивающих равномерную сходимость ряда (1) в любом разумном смысле. Приведем соответствующие определения для числовых рядов.

ОПРЕДЕЛЕНИЕ 1. Пусть ограниченное множество $U \subset \mathbb{Z}^{2}$. Тогда скажем, что $U \in A_{3}$, если из того, что точка $\left(k_{1}, k_{2}\right) \in U$ вытекает, что и весь целочисленньй параллелепипед $\left[-\left|k_{1}\right|,\left|k_{1}\right|\right] \times\left[-\left|k_{2}\right|,\left|k_{2}\right|\right] \cap \mathbb{Z}^{2} \subseteq U$.

ОПРЕДЕЛЕНИЕ 2. БУдем говорить, что числовой ряд $\sum_{m, n \in \mathbb{Z}} a_{m, n} u$-сxо$\partial u m c я \kappa$ числу $\alpha$, если для любого $\varepsilon>0$ существует такое $N$, что для любого множества $U \in A_{3}$, удовлетворяющего условию $\left\{(m, n) \in Z^{2}:|(m, n)| \leqslant N\right\} \subseteq U$ имеем

$$
\left|\sum_{(m, n) \in U} a_{m, n}-\alpha\right|<\varepsilon,
$$

где $|(m, n)|=\left(m^{2}+n^{2}\right)^{1 / 2}$.

Ясно, что если двойной числовой ряд $u$-сходится, то он сходится и по квадратам, и по Прингсхейму, и по кругам, и вообще в любом смысле из рассматривавшихся до сих пор. Необходимо отметить, что определение $u$-сходимости было введено $\Phi . \Gamma$. Арутюняном [3], [4] в связи с задачей представления функций кратными тригонометрическими рядами.

В статье [5] автором были установлены такие результаты (мы вновь приводим их только для двумерного случая и для непрерывных функций).

Tеорема Г. Если функиия $f(x, y) \in C\left(T^{2}\right) u \omega(f, \delta)=o\left(\delta^{1 / 2}\right) n p u \delta \rightarrow+0$, то ряд Фурье функиии $f(x, y)$ равномерно и-сходится.

Теорема Д. Существует функиия $f(x, y) \in C\left(T^{2}\right)$ такая, что $\omega(f, \delta)=O\left(\delta^{1 / 2}\right)$ при $\delta \rightarrow+0$, но ряд Фурье функиии $f(x, y)$ u-расходится всюду.

Таким образом, видно, что в нашей ситуации сходимость по кругам является, в определенном смысле, одной из наихудших. В то же время ясно, что, например, условиям теорем В и $\Gamma$ не удовлетворяет функция $f(x, y) \equiv f(x) \in \operatorname{Lip}(1 / 3)$, а ее ряд Фурье очевидно $u$-сходится. Поэтому представляет интерес получение утверждения, аналогичного теореме $\Gamma$, но выраженного в терминах частных и смешанного модулей непрерывности.

В данной статье будет установлен такой результат. 
TеОРема. Пусть функиии $\omega_{i}(\delta), i=1,2,3,4$, являются строго монотонными модулями непрерывности,

$$
\begin{aligned}
& \lim _{\delta \rightarrow+0} \omega_{i}(\delta) \ln \frac{1}{\delta}=0 \quad \text { npu } \quad i=1,2 ; \\
& \int_{0}^{\delta} \frac{\omega_{i}(t)}{t} d t=O\left(\omega_{i}(\delta)\right) \quad n p u \quad \delta \rightarrow+0 \quad \partial \Omega s \quad i=3,4 \\
& \lim _{\delta \rightarrow+0} \frac{\omega_{3}(\delta) \omega_{4}(\delta)}{\delta^{1 / 2}}=0,
\end{aligned}
$$

а функция $f(x, y) \in C\left(T^{2}\right)$ такова, что $\omega_{1}(f, \delta) \leqslant C \omega_{1}(\delta), \omega_{2}(f, \delta) \leqslant C \omega_{2}(\delta)$ $u \omega\left(f, \delta_{1}, \delta_{2}\right) \leqslant C \omega_{3}\left(\delta_{1}\right) \omega_{4}\left(\delta_{2}\right)$ nри всех $\delta, \delta_{1}, \delta_{2} \in(0,2 \pi)$.

Тогда двойной тригонометрический ряд Фурье функиии $f(x, y)$ равномерно и-сходится.

История возникновения условия (3) и других сходных с ним условий подробно изложена в статье П. Л. Ульянова [6].

Заметим, что невозможность ослабления условия (2) в теореме следует из одномерных соображений, а условия (4) - из теоремы Д. Данная теорема может быть обобщена и на случай $p \in[1, \infty), p \neq 2$, с использованием тех же соображений, что были применены автором в статье [5] при доказательстве теоремы $\Gamma$.

Отметим также, что из приведенной выше теоремы вытекает, что если $f(x, y)=g(x) h(y)$, где $\omega(g, \delta)=o\left(\delta^{1 / 4}\right), \omega(h, \delta)=O\left(\delta^{1 / 4}\right)$, то ряд Фурье функции $f(x, y)$ равномерно сходится по кругам. Вопрос об окончательности последнего утверждения остается открытым.

2. Доказательство теоремы. Нам понадобятся некоторые обозначения. Если $\mathbf{x}, \mathbf{y} \in \mathbb{R}^{2}$, то скажем, что $\mathbf{x} \geqslant \mathbf{y}(\mathbf{x}>\mathbf{y})$, если $x_{1} \geqslant y_{1}\left(x_{1}>y_{1}\right)$ и $x_{2} \geqslant y_{2}$ $\left(x_{2}>y_{2}\right)$.

Если функция $f(x, y) \in C\left(T^{2}\right)$ и $\nu=0,1, \ldots$, то обозначим через $W(\nu, 1, f)(x, y)$ средние Валле-Пуссена ряда Фурье функции $f(x, y)$ по переменной $x$ порядка $2^{\nu}$, т.е. $W(0,1, f)=S_{0}(1, f)$ и

$$
W(\nu, 1, f)=\frac{S_{2^{\nu-1}}(1, f)+\cdots+S_{2^{\nu}-1}(1, f)}{2^{\nu-1}}=2 \sigma_{2^{\nu}-1}(1, f)-\sigma_{2^{\nu-1}-1}(1, f)
$$

при $\nu>0$, где

$$
\sigma_{r}(1, f)(x, y)=\frac{1}{\pi} \int_{T} f(x+t, y) K_{r}(t) d t
$$

a

$$
K_{r}(\tau)=\frac{1}{r+1} \cdot \frac{\sin ^{2}(r+1) \tau / 2}{2 \sin ^{2} \tau / 2}
$$

- ядро Фейера. Аналогично определяются и $W(\nu, 2, f)$ - средние Валле-Пуссена по переменной $y$.

Далее, если $j=1$ или $j=2$, то положим $\Gamma(1, j, f)=W(0, j, f)$ и $\Gamma(r, j, f)=$ $W(r-1, j, f)-W(r-2, j, f)$ при $r=2,3, \ldots$. Если $\mathbf{n}=\left(n_{1}, n_{2}\right) \in \mathbb{Z}^{2} \cap[0,+\infty)^{2}$, то обозначим через

$$
G(\mathbf{n}, f)=\Gamma\left(n_{2}, 2, \Gamma\left(n_{1}, 1, f\right)\right)
$$

и

$$
B_{\mathbf{n}}=\left[2_{-}^{n_{2}-1}, 2^{n_{2}}\right] \times\left[2_{-}^{n_{1}-1}, 2^{n_{1}}\right],
$$


где $2_{-}^{\alpha}=2^{\alpha}$ при $\alpha \geqslant 0$ и $2_{-}^{\alpha}=0$ при $\alpha<0$.

Теперь, если $U \in A_{3}$, то положим

$$
\tau_{U}=\tau_{U}(f, x, y)=\sum_{\mathbf{n}=\left(n_{1}, n_{2}\right) \geqslant \mathbf{1}: B_{\mathbf{n}-\mathbf{2}} \cap U \neq \varnothing} G(\mathbf{n}, f)(x, y)
$$

и

$$
S_{U}(f)=S_{U}(f, x, y)=\sum_{\mathbf{k} \in U} a_{\mathbf{k}}(f) e^{i\left(k_{1} x+k_{2} y\right)},
$$

где $\left\{a_{\mathbf{k}}(f)\right\}_{\mathbf{k} \in \mathbb{Z}^{2}}$ - это коэффициенты Фурье функции $f(x, y)$ по двойной тригонометрической системе.

ЛЕмма 1. При $\mathbf{k} \in U$ имеем $a_{\mathbf{k}}\left(\tau_{U}\right)=a_{\mathbf{k}}(f)$.

Это утверждение в более общей ситуации было доказано автором в статье [5].

СлЕДСтвИЕ [5]. Если $U \in A_{3}$, то справедлива оиенка

$$
\left\|f-S_{U}(f)\right\| \leqslant\left\|f-\tau_{U}\right\|+\sum_{\mathbf{n} \geqslant \mathbf{1}: B_{\mathbf{n}-\mathbf{2}} \cap U \neq \varnothing}\left\|G(\mathbf{n}, f)-S_{U}(G(\mathbf{n}, f))\right\| .
$$

ЗАмечАНИЕ. Отметим, что если

$$
\varphi(x)=\frac{1}{2 \pi} \int_{T} f(x, y) d y
$$

то $\omega(\varphi, \delta) \leqslant \omega_{1}(f, \delta) \leqslant \omega_{1}(\delta)$ при $\delta>0$, а потому ряд Фурье функции $\varphi(x)$ равномерно сходится по признаку Дини-Липшица. Аналогично обстоит дело и для функции

$$
\psi(y)=\frac{1}{2 \pi} \int_{T} f(x, y) d x .
$$

Поэтому, не ограничивая общности, мы будем считать в дальнейшем, что у функции $f(x, y)$ коэфффициенты $a_{m, n}=0$ при $\min (|m|,|n|)=0$, т.е. что $G((1, k), f) \equiv 0$ и $G((k, 1), f) \equiv 0$ при всех $k$.

ЛЕмма 2. В предположсения теоремы справедлива оиенка

$$
\left\|G\left(\left(n_{1}, n_{2}\right), f\right)\right\| \leqslant C(f) \omega_{3}\left(2^{-n_{1}}\right) \omega_{4}\left(2^{-n_{2}}\right)
$$

npu $n_{1}>1 u n_{2}>1$.

ДоКАЗАТЕЛЬСтво. Пусть при $n_{1}>1$ и $n_{2}>1 T\left(n_{1}, n_{2}, f\right)(x, y)$ - это функция, осуществляющая наилучшее приближение "углом" порядка $\left(2_{-}^{n_{1}-3}, 2_{-}^{n_{2}-3}\right)$. Тогда по теореме М. К. Потапова [7]

$$
\begin{aligned}
\left\|G\left(\left(n_{1}, n_{2}\right), f\right)\right\| & =\left\|G\left(\left(n_{1}, n_{2}\right), f-T\left(n_{1}, n_{2}, f\right)\right)\right\| \\
& \leqslant C\left\|f-T\left(n_{1}, n_{2}, f\right)\right\| \leqslant C(f) \omega_{3}\left(2^{-n_{1}}\right) \omega_{4}\left(2^{-n_{2}}\right),
\end{aligned}
$$

что и требовалось доказать. 
Лемма 3. Пусть множсество $U \in A_{3}$ и для некоторого $\mathbf{n} \in \mathbb{Z}^{2} \cap(0,+\infty)^{2}$ имеем

$$
U \subseteq\left[-2^{n_{1}}, 2^{n_{1}}\right] \times\left[-2^{n_{2}}, 2^{n_{2}}\right]
$$

Тогда норма оператора

$$
\left\|S_{U}\right\|_{C \rightarrow C} \leqslant C 2^{\min \left(n_{1}, n_{2}\right) / 2}\left(\max \left(n_{1}, n_{2}\right)-\min \left(n_{1}, n_{2}\right)+1\right) .
$$

Данная лемма получена в [5] как непосредственное следствие из одного результата А. А. Юдина и В.А. Юдина [8].

Перейдем теперь непосредственно к доказательству теоремы. Пусть $U \in A_{3}$. Тогда по следствию и замечанию имеем

$$
\left\|f-S_{U}(f)\right\| \leqslant\left\|f-\tau_{U}\right\|+\sum_{\mathbf{n}>\mathbf{1}: B_{\mathbf{n}-\mathbf{2}} \cap U \neq \varnothing}\left\|G(\mathbf{n}, f)-S_{U}(G(\mathbf{n}, f))\right\| .
$$

Пусть

$$
F_{U}=\left\{\mathbf{n}=\left(n_{1}, n_{2}\right)>\mathbf{1}: B_{\mathbf{n}-\mathbf{2}} \cap U \neq \varnothing\right\}
$$

и

$$
N=N(U)=\max \left\{r:(r, r) \in F_{U}\right\}
$$

Тогда (см. лемму 2 и прямую теорему теории приближений)

$$
\begin{aligned}
\left\|f-\tau_{U}\right\| \leqslant & \left\|f-\sum_{\mathbf{n}: \mathbf{n} \leqslant \mathbf{N}} G(\mathbf{n}, f)\right\|+\sum_{\mathbf{n}>\mathbf{1}: \max \left(n_{1}-N, n_{2}-N\right)>0}\|G(\mathbf{n}, f)\| \\
\leqslant & \|f-W(N-1,2, W(N-1,1, f))\| \\
& +C(f)\left(\sum_{n_{1}=N+1}^{\infty} \sum_{n_{2}=2}^{\infty} \omega_{3}\left(2^{-n_{1}}\right) \omega_{4}\left(2^{-n_{2}}\right)\right. \\
& \left.+\sum_{n_{1}=2}^{\infty} \sum_{n_{2}=N+1}^{\infty} \omega_{3}\left(2^{-n_{1}}\right) \omega_{4}\left(2^{-n_{2}}\right)\right) \\
\leqslant & \|f-W(N-1,2, W(N-1,1, f))\|+C\left(f, \omega_{4}\right) \int_{0}^{2^{-N}} \frac{\omega_{3}(t)}{t} d t \\
& +C\left(f, \omega_{3}\right) \int_{0}^{2^{-N}} \frac{\omega_{4}(t)}{t} d t \rightarrow 0
\end{aligned}
$$

при $N \rightarrow \infty$. Теперь рассмотрим

$$
\begin{aligned}
\sum_{\mathbf{n} \in F_{U}}\left\|G(\mathbf{n}, f)-S_{U}(G(\mathbf{n}, f))\right\|=\sum_{\mathbf{n} \in F_{U}: n_{1} \leqslant n_{2}}\left\|G(\mathbf{n}, f)-S_{U}(G(\mathbf{n}, f))\right\| \\
+\sum_{\mathbf{n} \in F_{U}: n_{2}<n_{1}}\left\|G(\mathbf{n}, f)-S_{U}(G(\mathbf{n}, f))\right\|=V_{1}+V_{2}
\end{aligned}
$$


Поскольку $V_{1}$ и $V_{2}$ оцениваются совершенно одинаково, оценим $V_{1}$. Имеем (см. леммы 2 и 3 )

$$
\begin{aligned}
V_{1} & \leqslant \sum_{n_{1}=2}^{N} \sum_{n_{2}=\nu\left(n_{1}\right)}^{\mu\left(n_{1}\right)}\|G(\mathbf{n}, f)\|\left(1+\left\|S_{U}\right\|_{C \rightarrow C}\right) \\
& \leqslant C(f) \sum_{n_{1}=2}^{N} \sum_{n_{2}=\nu\left(n_{1}\right)}^{\mu\left(n_{1}\right)} \omega_{3}\left(2^{-n_{1}}\right) \omega_{4}\left(2^{-n_{2}}\right)\left(n_{2}-n_{1}+1\right) 2^{n_{1} / 2},
\end{aligned}
$$

где при любом $n_{1}$ имеем $\mu\left(n_{1}\right) \geqslant \nu\left(n_{1}\right) \geqslant n_{1}$ и $\nu\left(n_{1}\right) \geqslant N-2$.

В статье Н. К. Бари и С.Б. Стечкина [9] было установлено, что для модулей непрерывности условие (3) эквивалентно существованию такого $C>1$, что

$$
\varliminf_{\delta \rightarrow+0} \frac{\omega(C \delta)}{\omega(\delta)}>1 .
$$

Поэтому из условия теоремы вытекает, что для некоторого $C>1$ имеем

$$
\varliminf_{\delta \rightarrow+} \frac{\omega_{4}(C \delta)}{\omega_{4}(\delta)}=\alpha>1 .
$$

Но тогда

$$
\frac{\omega_{4}\left(2^{k_{0}} \delta\right)}{\omega_{4}(\delta)} \geqslant \beta^{k_{0}}>1
$$

для некоторых $k_{0}$ и $\beta$ при всех $\delta \in(0,1)$. Поэтому

$$
\begin{aligned}
& \sum_{n_{2}=\nu\left(n_{1}\right)}^{\mu\left(n_{1}\right)} \omega_{4}\left(2^{-n_{1}}\right)\left(n_{2}-n_{1}+1\right) \\
& \quad \leqslant \beta^{k_{0}} \omega_{4}\left(2^{-\nu\left(n_{1}\right)}\right)\left(\nu\left(n_{1}\right)-n_{1}+1\right) \sum_{k=0}^{\infty} \beta^{-k} \frac{k+\nu\left(n_{1}\right)-n_{1}+1}{\nu\left(n_{1}\right)-n_{1}+1} \\
& \quad \leqslant \beta^{k_{0}} \omega_{4}\left(2^{-\nu\left(n_{1}\right)}\right)\left(\nu\left(n_{1}\right)-n_{1}+1\right) \sum_{k=0}^{\infty} \beta^{-k}(k+1) \\
& \quad=C\left(\omega_{4}\right) \omega_{4}\left(2^{-\nu\left(n_{1}\right)}\right)\left(\nu\left(n_{1}\right)-n_{1}+1\right) \\
& \quad \leqslant C\left(\omega_{4}\right) \omega_{4}\left(2^{-N}\right)\left(N-n_{1}+1\right) \beta^{-\left(\nu\left(n_{1}\right)-N\right)}\left(1+\frac{\nu\left(n_{1}\right)-N}{N-n_{1}+1}\right) \\
& \quad \leqslant C\left(\omega_{4}\right) \omega_{4}\left(2^{-N}\right)\left(N-n_{1}+1\right) .
\end{aligned}
$$

Отсюда (см. (8) и (9))

$$
V_{1} \leqslant C\left(f, \omega_{4}\right) \omega_{4}\left(2^{-N}\right) \sum_{n=2}^{N} \omega_{3}\left(2^{-n}\right) 2^{n / 2}(N-n+1) .
$$


Отметим, что в статье Н. К. Бари и С. Б. Стечкина [9] было также доказано, что из условия (3) вытекает существование такого $\gamma \in(0,1)$, что функция $t^{-\gamma} \omega_{4}(t)$ почти возрастает, т.е. существует такое $R>0$, что если $x<y$, то

$$
\frac{\omega_{4}(x)}{x^{\gamma}} \leqslant R \frac{\omega_{4}(y)}{y^{\gamma}}
$$

Пусть теперь задано некоторое $\varepsilon>0$. Фиксируем $k$ так, что (см. (4))

$$
\omega_{3}\left(2^{-n}\right) \omega_{4}\left(2^{-n}\right) \leqslant \frac{\varepsilon}{2 C\left(f, \omega_{4}\right) R D+1} 2^{-n / 2}
$$

при $n>k$, где

$$
D=\sum_{r=0}^{\infty} 2^{-r \gamma}(r+1)
$$

Заметим, что для достаточно больших $N$ имеем

$$
C\left(f, \omega_{4}\right) \omega_{4}\left(2^{-N}\right) \sum_{n=2}^{k} \omega_{3}\left(2^{-n}\right) 2^{n / 2}(N-n+1)<\frac{\varepsilon}{2} .
$$

Наконец (см. (11)),

$$
\begin{aligned}
& C\left(f, \omega_{4}\right) \omega_{4}\left(2^{-N}\right) \sum_{n=k+1}^{N} \omega_{3}\left(2^{-n}\right) 2^{n / 2}(N-n+1) \\
& \quad=C\left(f, \omega_{4}\right) 2^{-N \gamma} \omega_{4}\left(2^{-N}\right) 2^{N \gamma} \sum_{n=k+1}^{N} \omega_{3}\left(2^{-n}\right) 2^{n / 2}(N-n+1) \\
& \leqslant C\left(f, \omega_{4}\right) 2^{-N \gamma} R \sum_{n=k+1}^{N} \omega_{3}\left(2^{-n}\right) \omega_{4}\left(2^{-n}\right) 2^{n(1 / 2+\gamma)}(N-n+1) \\
& \quad \leqslant \frac{\varepsilon}{2 D} 2^{-N \gamma} \sum_{n=k+1}^{N} 2^{n \gamma}(N-n+1) \leqslant \frac{\varepsilon}{2 D} \sum_{r=0}^{\infty} 2^{-r \gamma}(r+1)=\frac{\varepsilon}{2} .
\end{aligned}
$$

Из формул (10), (12) и (13) вытекает, что при достаточно больших $N$ имеем $V_{1}<\varepsilon$. Поскольку $V_{2}$ оценивается аналогично, из формул (5)-(7) следует утверждение теоремы.

Московский государственный университет

Поступило

им. М. В. Ломоносова

28.03 .95

E-mail: mid@nw.math.msu.su

\section{СПИСОК ЦИТИРОВАННОЙ ЛИТЕРАТУРЫ}

1. ЖКижиашвили Л. В. // УМН. 1973. Т. 28. №2. С. 65-119. 2. Алимов Ш. А., Ильин В.А. // Дифференц. уравнения. 1971. Т. 7. №4. С. 670-710. 3. Арутюнян Ф. Г. // Докл. АН АрмССР. 1977. Т. 64. № 2. С. 72-76. 4. Арутюнян Ф.Г. // Матем. сб. 1985. Т. 126. № 2. С. 267-285. 5. Дьяченко М.И. // Изв. РАН. Сер. матем. 1995. Т. 59. № 2. С. 128-142. 6. Ульянов П. Л. // Докл. РАН. 1993. Т. 333. № 2. С. 157-160. 7. Потапов М. К. // Тр. МИАН. 1972. Т. 117. С. 256-291. 8. Юдин А. А., Юдин В. А. // Матем. заметки. 1977. Т. 22. № 3. С. 381-394. 9. Бари Н. К., Стечкин С. Б. // Тр. ММО. 1956. Т. 5. С. 485-522. 